\title{
A Possible Molecular Counterpart To The G359.54+0.18 Non-Thermal Filaments
}

\author{
John Bally \\ AT\&T Bell Laboratories \\ Holmdel, New Jersey \\ Farhad Yusef-Zadeh ${ }^{1,2}$ and J.M. Hollis \\ Space Data and Computing Division \\ NASA/Goddard Space Flight Center
}

\begin{abstract}
We present new 30" resolution $\mathrm{J}=2-1{ }^{12} \mathrm{CO}$ observations of the molecular gas located close to a group of non-thermal radio emitting filaments recently detected near the Galactic center (Bally and Yusef-Zadeh, 1989). The new data provide some support for the association of the non-thermal filaments G359.54+0.18 with the projected edge of a molecular cloud.
\end{abstract}

\section{Introduction}

High angular resolution observations with the VLA have led to the discovery of several groups of unusual, filamentary, thermal and non-thermal radio structures lying within one degree of the Galactic center (Yusef-Zadeh, Morris, and Chance 1984; Liszt 1985; Yusef-Zadeh 1986; Yusef-Zadeh and Morris 1987). The brightest non-thermal filaments are seen to cut across the Galactic plane in the Continuum Arc at $l=0.18^{\circ}$ and near Sgr C. A group of thermal filaments extend from the high latitude side of the Continuum Arc to towards the plane near Sgr A. A number of fainter "threads" of radio emission can also be seen towards this region.

Brown and Liszt (1984) and Bally et al. (1988) have drawn attention to the possible association of these thermal and non-thermal filaments with the edges of molecular clouds lying along the line-of-sight. The molecular gas and the filaments have similar distributions in the sky; both class of objects are confined to the lowlatitude portion of the Galactic plane and show the most intense emission at positive longitudes. The association between the "arched" thermal filaments (G0.10+0.08) and the $\mathrm{V}_{\mathrm{LSR}} \approx-30 \mathrm{~km} \mathrm{~s}^{-1}$ molecular cloud is reasonably secure since this molecular cloud and the filaments have a similar distribution on the sky and the CO and CS radial velocities have the same value as the radio recombination line velocities (Serabyn and Güsten 1987). Observations of interaction between the thermal and non-thermal filaments may provide further indirect evidence for an association of the non-thermal emission with the molecular cloud distribution. There are molecular clouds in the vicinity of both the Arc and $\mathrm{Sgr} \mathrm{C}$ whose edges lie close to the non-thermal structures. However, the large concentration of molecular gas in these directions makes a chance

\footnotetext{
1 NRC Resident Research Associate

2 On leave from the Department of Physics and Astronomy. Northwestern University.
} 
superposition of a cloud edge and a group of filaments possible.

The recent detection of a new group of non-thermal filaments in G359.54+0.18 $15^{\prime}$ north of Sgr C by Bally and Yusef-Zadeh (1989) in a region located above the highly confused region of the Galactic plane provides a new opportunity to investigate the possible relationship between filaments and molecular gas. Indeed, observations obtained with a 100" beam in the $100 \mathrm{GHz}$ lines of $\mathrm{CO}$ and $\mathrm{CS}$ indicate that G359.54+0.18 lies near the projected edge of a peculiar molecular cloud centered at $\mathrm{V}_{\mathrm{LSR}} \approx-140 \mathrm{~km} \mathrm{~s}^{-1}$ (Figure 1). If a physical association is confirmed, this will place a strong constraint on models of the nature and origin of this enigmatic new class of structure in the interstellar medium. Here, we present new observations of this region with a nearly 4-fold improvement in angular resolution.

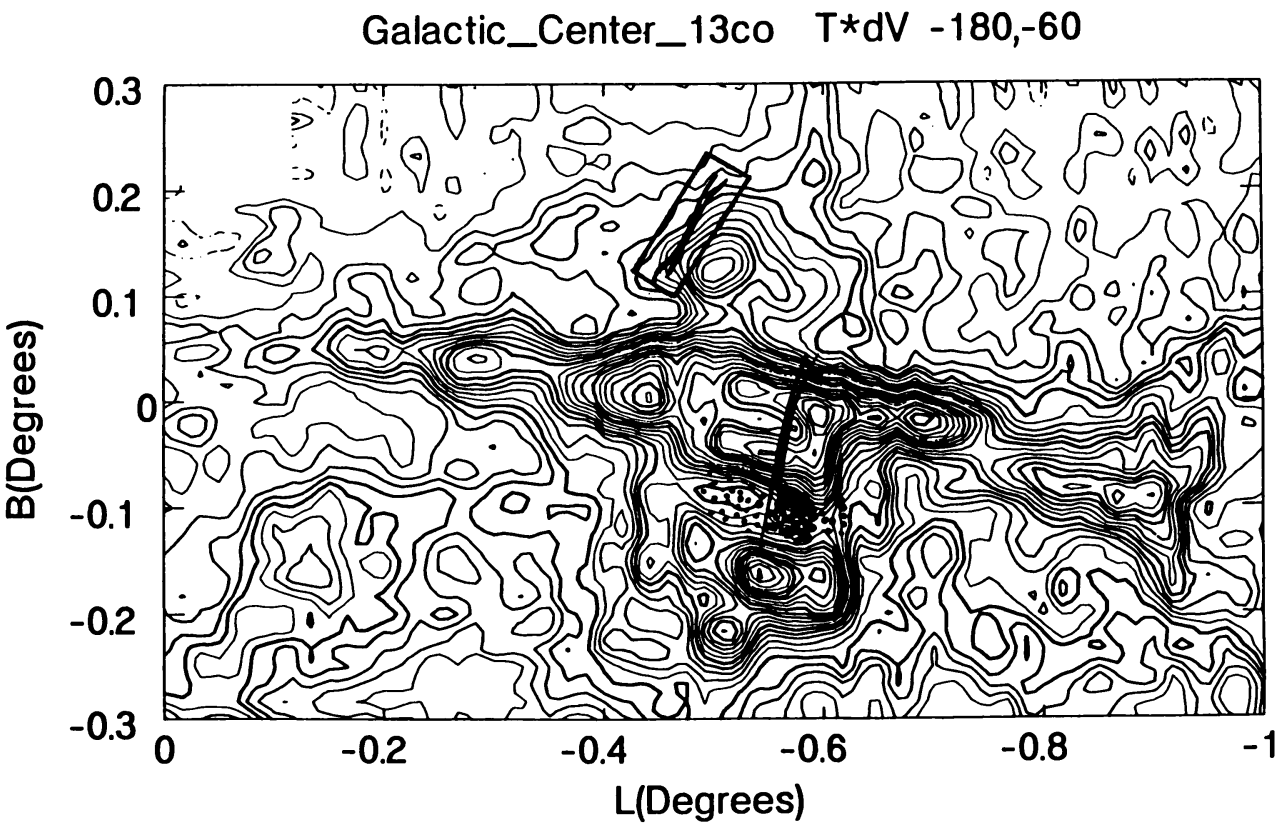

Figure 1: A contour map of a 1 degree long portion of the Galactic plane from $1=359^{\circ}$ to $l=0^{\circ}$ in ${ }^{13} \mathrm{CO}$ emission integrated from $\mathrm{V}_{\mathrm{LSR}}=-180$ to $-60 \mathrm{~km} \mathrm{~s}^{-1}$. Contour levels are set at $10 \mathrm{Kkm} \mathrm{s}^{-1}$ intervals and range from $-10 \mathrm{Kkm} \mathrm{s}^{-1}$ to $120 \mathrm{Kkm} \mathrm{s}^{-1}$. The location of the Sgr C (near center) and G359.54+0.18 (near top) filaments are indicated. The rectangle near the top illustrates the extent and orientation of the map shown in Figure 3. The center of the Galaxy ( $\mathrm{Sgr} \mathrm{A}$ ) lies about 2 arc-minutes to the right and below $(0,0)$. From Bally and Yusef-Zadeh (1989). 


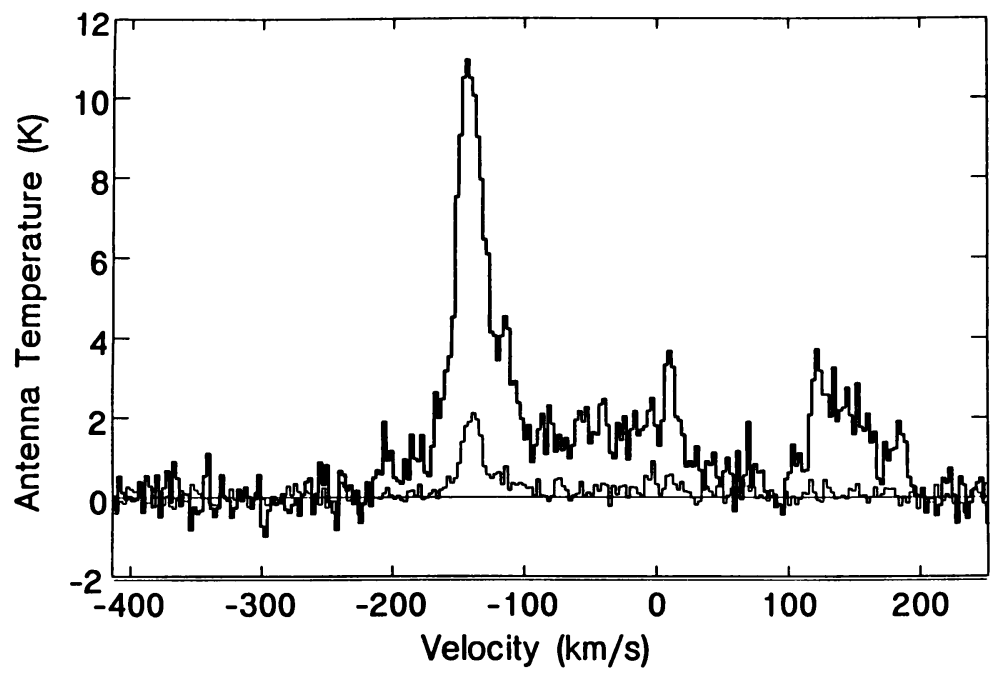

Figure 2: $\mathrm{J}=2-1{ }^{12} \mathrm{CO}$ (bold) and ${ }^{13} \mathrm{CO}$ (light) spectra at the center of the -140 $\mathrm{km} \mathrm{s}^{-1}$ molecular cloud at $\alpha(1950)=17^{\mathrm{h}} 40^{\mathrm{m}} 55.6^{\prime \prime}, \quad \delta(1950)=-29^{\circ} 14^{\prime} 00^{\prime \prime}$, which corresponds to $(2.1,1.4)$ in Figure 3 . Note the $200 \mathrm{~km} \mathrm{~s}^{-1}$ velocity range of the ${ }^{12} \mathrm{CO}$ emission between $\mathrm{V}_{\mathrm{LSR}}=-200$ and $0 \mathrm{~km} \mathrm{~s}^{-1}$.

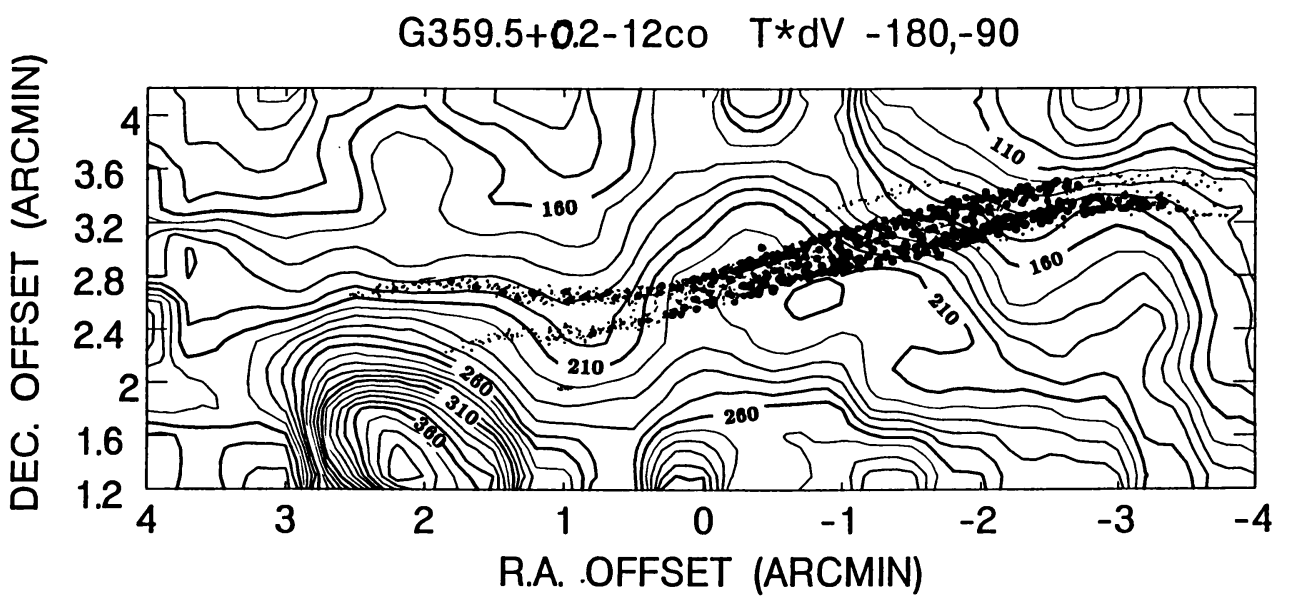

Figure 3: A contour map showing the distribution of ${ }^{12} \mathrm{CO}$ emission integrated from $\mathrm{V}_{\mathrm{LSR}}=-180$ to $-90 \mathrm{~km} \mathrm{~s}^{-1}$ which corresponds to the strongest spectral feature in Figure 2. Note that this figure is in equatorial co-ordinates while Figure 1 is in Galactic co-ordinates. Map co-ordinates are arc-minute offsets from a point at $\alpha(1950)=17^{\mathrm{h}} 40^{\mathrm{m}} 45.9^{\mathrm{s}}, \delta(1950)=-29^{\circ} 15^{\prime} 26^{\prime \prime}$. The location, morphology, and structure of the non-thermal filaments is shown by the dotted pattern. Contour levels are displayed at at $10 \mathrm{Kkm} \mathrm{s}^{-1}$ intervals and range from 70 to $390 \mathrm{Kkm} \mathrm{s}^{-1}$. 


\section{Observations And Results}

We obtained $230 \mathrm{GHz} \mathrm{J}=2-1{ }^{12} \mathrm{CO}$ and $220 \mathrm{GHz}{ }^{13} \mathrm{CO}$ observations using the NRAO 12-meter telescope (30" beam) during May 1988. We mapped an 8 by 3 arcminute field-of-view containing the radio filaments on a 30 " grid with some data on a $15 "$ grid. Figure 2 shows spectra taken at the peak of the $-140 \mathrm{~km} \mathrm{~s}^{-1}$ cloud which is located at the eastern end of the continuum filaments. The ${ }^{12} \mathrm{CO}$ spectrum has a broad component of emission which fills in the spectrum between the $-140 \mathrm{~km} \mathrm{~s}^{-1}$ cloud and the foreground/background emission from the Galactic plane near 0 $\mathrm{km} \mathrm{s}^{-1}$. Also seen is emission from gas near $+135 \mathrm{~km} \mathrm{~s}^{-1}$ which is produced by the so-called "expanding molecular ring" which can be traced over several degrees of sky. Figure 3 shows a map of the $\mathrm{J}=2-1{ }^{12} \mathrm{CO}$ emission integrated over a velocity range of $90 \mathrm{~km} \mathrm{~s}^{-1}$ from $\mathrm{V}_{\mathrm{LSR}}=-180$ to $-90 \mathrm{~km} \mathrm{~s}^{-1}$ in which the molecular cloud near the filament is clearly defined above the background emission.

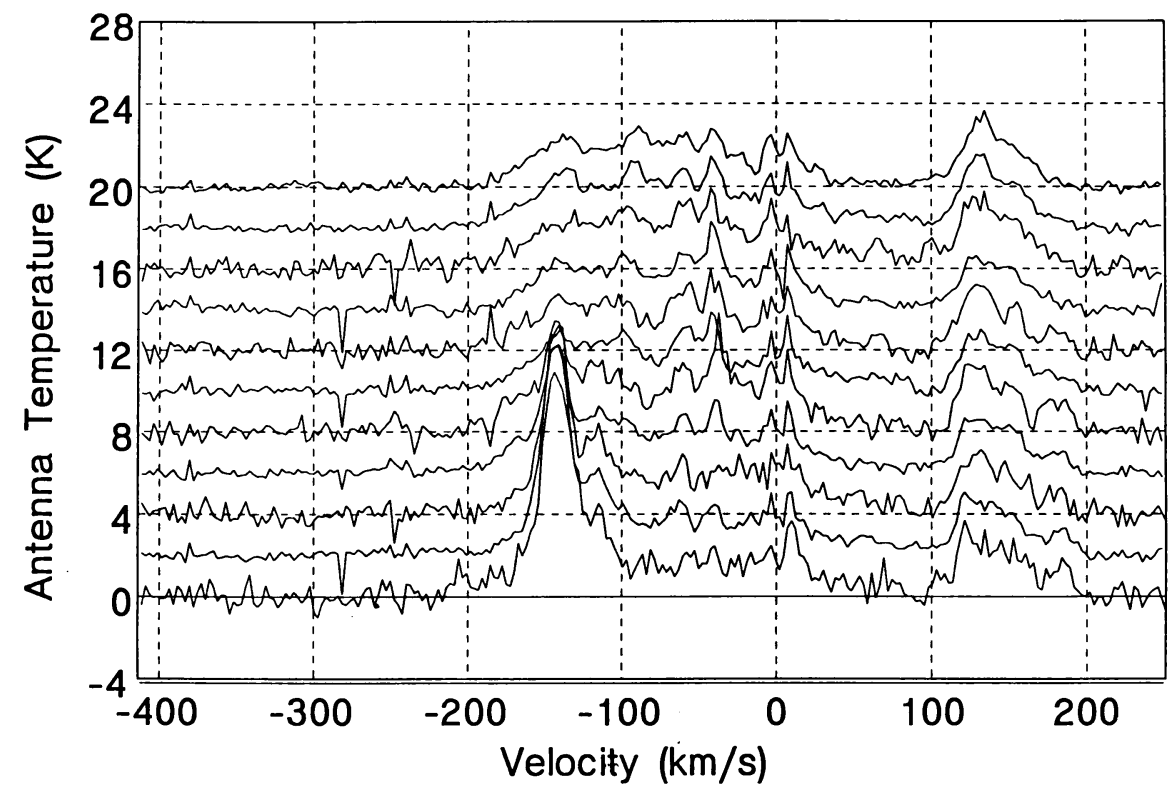

Figure 4: Spectra taken along a south-to-north strip cutting across the the filaments at $\alpha(1950)=17^{\mathrm{h}} 40^{\mathrm{m}} 55.6^{\prime \prime}\left(\Delta \alpha=2.1^{\prime}\right)$ separated by $15^{\prime \prime}$. The bottom spectrum is at the position of Figure 2. The south-to-north intensity drop near -140 $\mathrm{km} \mathrm{s}^{-1}$ is less dramatic further west.

Much small scale structure can be seen in the ${ }^{12} \mathrm{CO}$ data which is not apparent in Figure 1. Evidence for a correlation between the distribution of the molecular gas and the filaments occurs between $\mathrm{V}_{\mathrm{LSR}} \approx-180$ and $-90 \mathrm{~km} \mathrm{~s}^{-1}$ where the edge of the brightest clump of gas in the mapped field of view coincides with the bend at the eastern end of the radio filament. Although, along most of the remaining length of the radio structure, there is little evidence for the coincidence with a well defined cloud edge, there is a decline in the $\mathrm{CO}$ emission from south-to-north across the filaments near the velocity of the $-140 \mathrm{~km} \mathrm{~s}^{-1}$ cloud. Inspection of Figure 3 shows that the CO 
emissivity in the velocity range $V_{L S R}=-180$ to $-90 \mathrm{~km} \mathrm{~s}^{-1}$ declines by about 100 $\mathrm{K} \mathrm{km} \mathrm{s}^{-1}$ over the length of the filament from the bottom half to the top half of the figure. Figure 4 shows spectra along a south-to-north strip cutting across the eastern end of the radio filaments.

\section{Discussion}

The new, high-angular-resolution data demonstrates, that on a 15 " scale, the ${ }^{12} \mathrm{CO}$ emission correlates weakly with the filamentary radio continuum structure. A correlation is most obvious at the eastern end of the filaments where it appears to bend around the core of the $-140 \mathrm{~km} \mathrm{~s}^{-1}$ cloud. The filaments lie on a large ${ }^{12} \mathrm{CO}$ gradient. Figure 2 demonstrates that the opacity of the ${ }^{12} \mathrm{CO}$ line is $\leq 5$ at most velocities. These observations are most sensitive to low column density molecular gas than the previous ${ }^{13} \mathrm{CO}$ data and mostly probe gas lying in front of the $\tau \sim 1$ surface. It may not be possible to see the interior of clouds along the line of sight. Since much of the structure observed in ${ }^{12} \mathrm{CO}$ along this line of sight may not belong to a single object but represent a variety of unrelated structures, source confusion may still be a problem in this portion of the sky in a thick species such as ${ }^{12} \mathrm{CO}$.

Since the gas density near cloud "edges" usually drops as a smooth function of distance, the position of the cloud edge will depend on the sensitivity of the observations and on the spectral line being used to probe the cloud. Our observations suggest that if there is a connection between clouds and filaments, the filaments exist near the apparent ${ }^{13} \mathrm{CO}$ boundary, and probably inside the ${ }^{12} \mathrm{CO}$ emitting region. Furthermore, the apparent coincidence between cloud edges and filaments may be mostly a large scale phenomenon occuring on scales of order arc-minutes.

Benford (1988) has produced a quantitative model for the production of nonthermal filaments by the motion of a dense molecular cloud through a magnetized medium (see also Morris and Yusef-Zadeh 1988). Bally et al. (1988) provide a qualitative description of how compression of the inter-cloud magnetic fields by a shock produced from cloud motion, can accelerate the local cosmic ray population by either conservation of the first adiabatic invariant or by the Fermi acceleration process. In-situ particle acceleration injects electrons into the magnetic field lines, causing an increase in the synchrotron emissivity. These processes imply that filament production may be driven by the motion of the dense component of the interstellar medium near the Galactic center. Further observations, hopefully aided by the discovery of more filaments, must be undertaken to prove the possible association of filaments with molecular clouds.

\section{References}

Bally, J. and Yusef-Zadeh, F. 1989, Ap.J., (January 15, in press).

Bally, J., Stark, A.A., Wilson, R.W., and Henkel, C. 1988, Ap.J., 324, 223.

Benford, G. 1988, Ap.J., (in press).

Brown , R.L. and Liszt, H.S. 1984, Ann.Rev.Astr.Ap., 22, 223.

Liszt, H.S. 1985, Ap.J. (Letters), 239, L65.

Morris, M. and Yusef-Zadeh, F. 1988 Ap.J. (submitted).

Serabyn, E. and Güsten, R. 1987, Astron.Astrophys., 184, 133.

Yusef-Zadeh, F. 1980, Ph.D Thesis, Columbia University.

Yusef-Zadeh, F., Morris, M., and Chance, D. 1984, Nature, 310 , 557.

Yusef-Zadeh, F. and Morris, F. 1987, Ap.J., 322, 721. 